\title{
RESEARCH IN JET MIXING OF COMPONENTS OF NUTRIENT MEDIA IN SMALL- SCALE PRODUCTION OF MICROBIAL PESTICIDES
}

\author{
Valentyna Krutyakova ${ }^{1}$, Vladislav Yaroshevsky ${ }^{1}$, Volodymyr Bulgakov ${ }^{2}$, Semjons Ivanovs ${ }^{3}$ \\ ${ }^{1}$ Engineering and Technology Institute "Biotechnica", Ukraine; \\ ${ }^{2}$ National University of Life and Environmental Sciences of Ukraine, Ukraine; \\ ${ }^{3}$ Latvia University of Life Sciences and Technologies, Latvia \\ semjons@apollo.lv
}

\begin{abstract}
The production of microbiological preparations for agricultural needs is often implemented on the basis of small and medium-tonnage regional production facilities. The most common technology used at such enterprises is submerged cultivation of microorganisms using the fermentation apparatus. The use of a nonstandard fermenter necessitated the creation of additional equipment and the development of a non-standard technology for preparation and sterilization of nutrient media - an automated technological line for the production of biological products - a fermentation complex of the modular type KFM-420. Analysis of the technological process shows that mixing the components and obtaining the finished products actually occurs inside the fermenter. A comparison of the efficiency of the standard scheme for mixing the components with jet mixing based on the experimental results, shows that the use of the jet mixer can reduce the mixing time more than by half. This led to proportional power consumption decrease from $60 \mathrm{Wh}$ for standard technique of nutrient components supplying to the fermenter and further mixture homogenizing by agitator to $25 \mathrm{Wh}$ for jet mixing of the components. There is substantiated the expediency of using jet mixing of components as the most energy-efficient technology for the preparation of nutrient media from liquid components that have undergone separate sterilisation.
\end{abstract}

Keywords: component, nutrient medium, mixer, jet mixing, thin-walled fermenter.

\section{Introduction}

Currently, the use of microbiological preparations to protect plants from diseases and pests is one of the main areas of practical implementation of agricultural biologisation methods in the world. The production of microbiological preparations for agricultural needs is often implemented on the basis of small and medium-tonnage regional production facilities [1]. The most common technology used at such enterprises is deep cultivation of microorganisms using the fermentation apparatus.

As the analysis of numerous investigations and tests of a wide class of industrial serial bioreactors for small-tonnage production of microbiological preparations for plant protection shows, their use leads to a significant increase in the operating costs [1-3].

As a result, this has an effect on increasing the production cost and reducing its competitiveness in the market. The way out of this situation is the use of non-standard energy-efficient fermentation apparatus. The Engineering and Technological Institute "Biotechnica" of NAAS investigated and constructed a family of thin-walled fermenters (the corresponding volumes: $501,1001,1801$ ) [3; 4] and developed for them a new technology for small-tonnage production of the plant protection products based on them $[1 ; 2]$

A distinctive feature of this development is the implementation of the technological processes of the pre-fermentation stage and the stage of cultivation of microorganisms at atmospheric pressure. The absence of a high overpressure in the apparatus causes a significantly lower level of loads on the internal surfaces, as compared to the standard industrial bioreactors, and it also makes it possible to remove these devices from the list of vessels operating under pressure. This, in its turn, significantly softens the requirements for the manufacture and operation of the fermenters. A distinctive feature of these devices is the small thickness of the walls. Therefore, the metal consumption for the structure of the thin-walled fermenters is much lower than for the industrial bioreactors of the same volume.

The purpose of the research is the development of a new structural and technological scheme and the substantiation of the parameters of jet mixing of the components of nutrient media in the production of microbiological products.

\section{Materials and methods}

Application of a non-standard fermenter necessitated the creation of additional equipment and the development of a non-standard technology for preparation and sterilisation of the nutrient media (NM) 
- an automated technological line for the production of biological products - a fermentation complex of the modular type KFM-420 [1-3].

Within the framework of the Fermentation Complex, the technology of separate sterilisation of NM components is implemented. Typically, this technique is used for thermolabile components of the $\mathrm{NM}$, thermal sterilisation of which is impossible [5, 6]. However, in this case the use of this technology is aimed at reducing the energy costs for sterilisation. Thermal sterilisation is carried out only by a nutrient medium concentrate (NMC), which ranges from $1 / 8$ to $1 / 3$ of the total volume of the NM. The solvent for the NMC is water, which makes up most of the NM. It is sterilized by ultraviolet radiation with low energy costs [3].

The complexity of the pre-fermentation stage of production caused certain shortcomings in the preparation of the NM. First, the issue of dosing the NMC to water remained unresolved, because both of these components of the medium are simultaneously prepared and distributed between the three fermenters. Second, for preparation of the NM, it was not enough simply to dilute the NMC with water inside the fermenter.

To obtain a homogeneous mixture, it is necessary additionally to mix the components with a fermenter stirrer, which leads to an increase in the duration of the process and energy consumption. The conducted investigations show that these shortcomings can be corrected by using jet mixing of the components during the preparation of the NM. Therefore, the main focus of this work is to determine a possibility to improve the process of preparing the NM, based on the use of jet mixing of the components, as well as the development of an apparatus of a simple design for implementation of this process.

The indicated technological difficulties are not unique and inherent only to the thin-walled fermenters. Therefore, the solution to the problem of increasing the energy efficiency for preparation of homogeneous mixtures of the NM from liquid and solid components is relevant for most industrial production of biological products of various directions.

We have made an analysis of the existing technology for preparation of media on the basis of an experimental sample of a microbiological product production line - the KFM-420 Fermentation Complex. In addition, a qualitative assessment is given of the effect from replacing the standard PS preparation technology by sequential feeding of the components into the fermenter for jet mixing in a separate apparatus. In order to determine the design of the mixer, analysis of the organisation of the jet processes, used in the preparation of suspensions for various industries, was performed.

Based on the generalization of the results of the analysis on the preparation technology of PS and jet mixing schemes, taking into account the requirements for the design of the apparatus for microbiological production conditions, we have developed a new design of the jet mixer.

The technology for the NM preparation on the equipment of an experimental sample of the fermentation complex provides for the presence of two successive stages. At the first stage, there is a separate preparation and sterilisation of two components of the medium - the NMC and water; at the second - mixing of the components and obtaining the NM [3]. The new technological scheme for the preparation of the NM, developed by us, is shown in Fig. 1a.

The preparation of the NMC and water at the first stage takes place in separate apparatuses. In this case the NMS, as noted above, is sterilised in a standard way in the steriliser 2: the medium is heated to a temperature of $120^{\circ} \mathrm{C}$ and kept for an estimated time. At the same time the water that is necessary for diluting the NMS is filtered; it undergoes UV treatment and accumulates in a special container.

The second stage begins with transportation of the NMC from the steriliser 2 to the thin-walled fermenters 1 . Then, the calculated amount of purified and disinfected water is added to the NMC inside the fermenter 1 , which is pumped by the pump 3 and dosed, using the flow meter 4 . To homogenise the obtained mixture, the mechanical mixer of the fermenter 5 is used. Mixing of the components occurs within 15-20 minutes.

The energy consumption for mixing the NMC with water in a ratio of 1: 3 for three fermenters of the complex with a working volume of 801 each is $0.18 \mathrm{kWh}$. It is worth adding that, in order to reduce quickly the temperature of the mixture to the temperature of cultivation, the water is pre-cooled 
$[1 ; 3]$, since the temperature of the $\mathrm{NMC}$, entering the fermenter, reaches $100{ }^{\circ} \mathrm{C}$, and the temperature of cultivation is within the range of $27-30^{\circ} \mathrm{C}$.

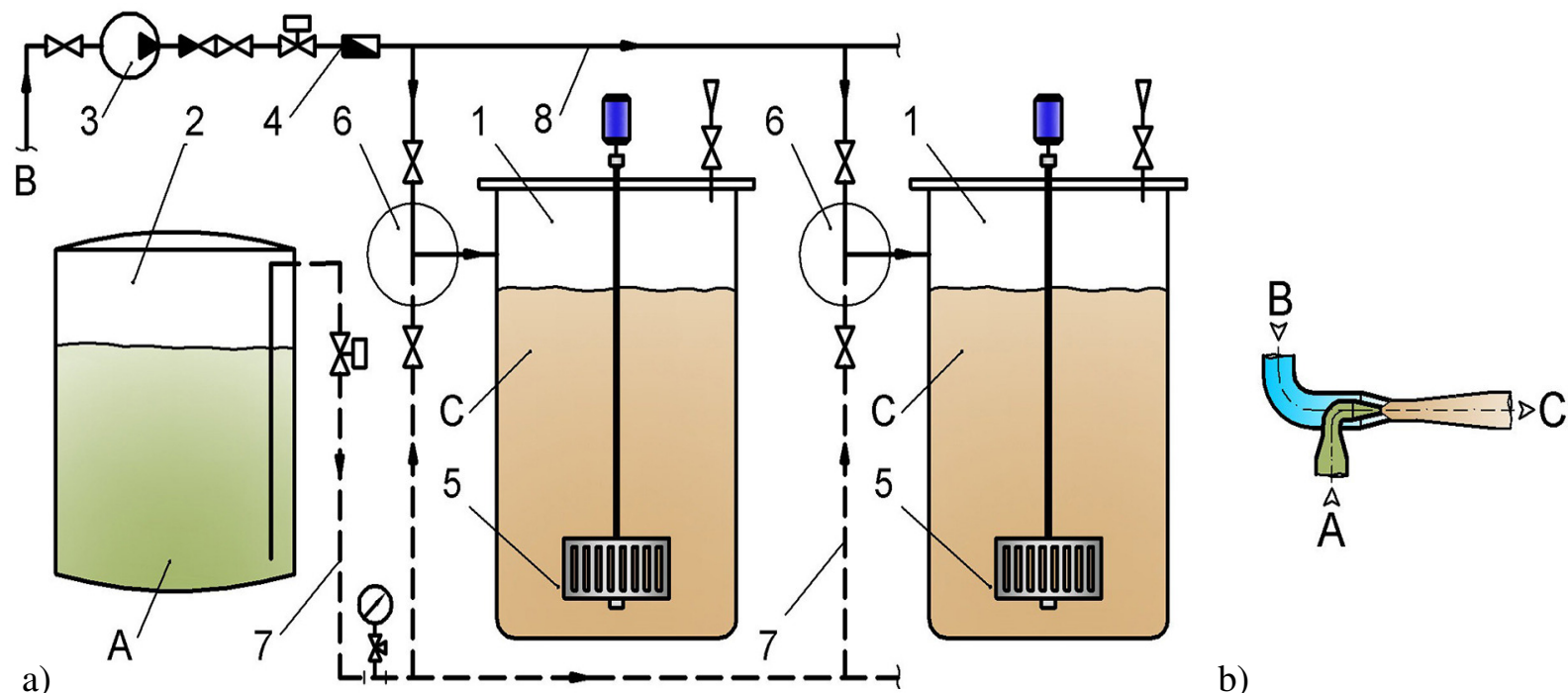

Fig. 1. Technological scheme for preparation of NM (a) and the scheme of jet mixing NMC with water (b): 1 - thin-walled fermenter; 2 - sterilizer; 3 - pump; 4 - flow meter; 5 - mixer; 6 - place for the jet mixer; 7 - NMC feed line; 8 - water supply line; A - NMC; B - water; $\mathrm{C}$ - finished PS

\section{Results and discussion}

Analysis of the technological process shows that mixing the components and obtaining the finished NM actually occurs inside the fermenter. Application of the jet mixing (Fig. $1 \mathrm{~b}$ ) will allow the main process to be moved from the fermenter to a separate apparatus - the jet mixer (JM). This will allow, with proper organisation of the jet processes and preliminary cooling of water, to supply to the fermenter the NM that is ready for inoculation.

As experience of using jet apparatus (JA) in various industries [7-9] indicates, intense heat and mass transfer between the flows should take place inside the mixer. Thus, at the outlet of the apparatus, it is possible to obtain a mixture with the required temperature, which does not need additional homogenisation using a mechanical fermenter stirrer. In addition, the replacement of the sequential supply of the NMC and water to the fermenter with a parallel supply to the JA should lead to a reduction in the duration of the process, and, consequently, to a reduction in the energy consumption for mixing. Besides, a characteristic feature of the JA, as a variety of the JM, is keeping constant proportions of the mixing liquids. That is why such devices often serve as dispensers. Consequently, in addition to the effects noted above, the use of the jet mixing scheme leads to simplification of the process of dispensing the NMC to water.

Since the JA for small flows of liquids have small dimensions [7], and they are also often easy to manufacture, it is purposeful to provide a separate apparatus for each fermenter. Proceeding from this, the most appropriate place for placing the JM is the region 6 in front of the entrance to the fermenter (Fig. 1 a).

In the process of determining the design peculiarities of the JM, it is necessary to take into account a number of specific requirements relating to the organisation of technological processes of microbiological productions. In particular, the design of the apparatus should prevent penetration of extraneous micro flora into the mixing liquids, but allow washing and easy dismantling [2]. These requirements can be taken into account in the case of using a collapsible design of the JM.

As a rule, the small dimensions of the JA allow, after its dismantling, to place them in the autoclave and carry out thermal sterilisation. For the JM, sterilisation may also be used in assembling by a flow-through method, using liquid disinfectants. The washing process may be similarly organised, only instead of disinfectants, detergents will be supplied to the apparatus. Thus, the use of a collapsible JM design, on the one hand, will allow washing and sterilisation of the apparatus using a flow-through method and appropriate agents, and, on the other hand, it will allow periodical 
dismantling of the JM, its disassembling into its components, washing them separately and further autoclaving together with other elements of the thin-walled fermenter strapping [2].

It should be noted that the JA is mainly manufactured directly for the needs of a particular hydraulic or aerodynamic system. The lack of standardized devices contributed to the emergence of a large number of modifications of the classic design of the JA. Thus, the analysis shows that at the basis of the collapsible structures of JA, as a rule, there are atypical elements: mixing chambers [10], non-standard tees $[11 ; 12]$, etc. Application of standard shaped elements of the pipeline systems for the development of the JS will greatly simplify not only its design, but also the method of connection to the existing fermenter strapping system. In our opinion, it is advisable to use shaped tees as such elements. The next step in the development of the design of the JS is to choose a mixing scheme. In accordance with the mixing scheme, the apparatus can be divided into two main groups: the flowthrough and the counterflow apparatus. In the counterflow JS mixing occurs in the oncoming flow, in the flow-through ones, in the concurrent one. At the moment it is customary to give preference to the counter-current mixing scheme [10]. However, it supposes the use of powerful superchargers, since mixing in the oncoming flow occurs with significant losses of energy. In addition, both flows should have approximately the same pressure. At the same time, within the framework of the Fermentation Complex, the NMC and water are transported under pressures that differ by almost an order of a magnitude. Therefore, it is expedient to organise mixing in the JS according to the flow pattern, which brings the mixer closer to the classic JA.

The through-flow JSs basically consist of three parts: a nozzle for introducing an active flow, a mixing chamber for the active and passive flows, and a diffuser for levelling the velocity field of the mixed flow. In accordance with the accepted location (6, Fig. 1 a), the mixers will be installed practically just next to the fermenter. Accordingly, there is no need to equalise the velocity field, and, consequently, to use a diffuser. Based on the generalisation of the presented data, we proposed a new design of a diffuserless type JS (Fig.2).

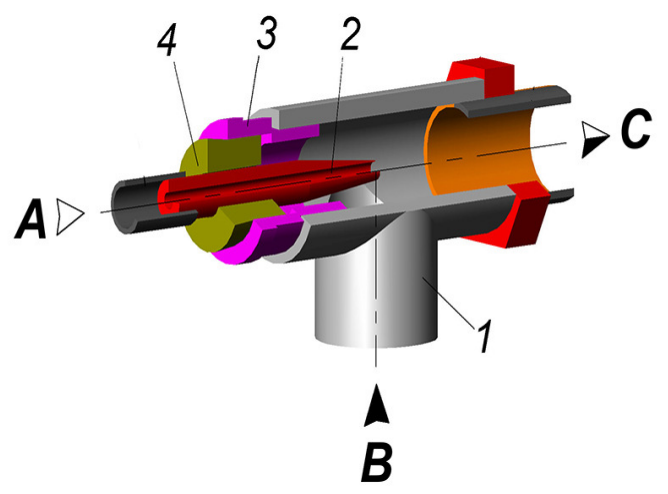

Fig. 2. Jet mixer of the nutrient media components of the diffuserless type: 1 - body (a shaped tee); 2 - nozzle; 3 - transitional pipe coupling; 4 - compactor; A - NMC;

$$
\mathrm{B} \text { - disinfected water; } \mathrm{C} \text { - finished NM }
$$

This JS consists of the nozzle 2, centred relative to the longitudinal axis of the housing 1 (tee) and fixed with the compactor (gasket) 4 inside a standard pipe transition 3 . The section of the nozzle 2 coincides with the axis of the passive flow - water.

The NMC will be supplied under the pressure of 1.5-2.0 bar, but to the pipe of the passive flow water will be supplied under the pressure of $0.2-0.5$ bar. If there is no diffuser, mixing of the components will begin in the tee, and will continue in the pipe, adjacent to the entrance to the fermenter. A comparison of the efficiency of the standard scheme for mixing the NM components with jet mixing (Table 1), based on the results of preliminary tests, shows that the use of the JS can reduce the mixing time practically by half.

This affects the reduction of energy consumption by more than 2 times. However, in the case of jet mixing, the final temperature of the mixture is $37^{\circ} \mathrm{C}$, which is almost by $10^{\circ} \mathrm{C}$ higher than the temperature that is necessary for sowing most microorganisms used in plant protection. At the same time, with a standard mixing scheme, the final temperature of the mixture corresponds to the sowing temperature. 
Comparison of the efficiency of the mixing schemes of the NM components, based on the results of preliminary tests

\begin{tabular}{|c|c|c|c|}
\hline $\begin{array}{c}\text { Scheme of mixing NM } \\
\text { components }\end{array}$ & $\begin{array}{c}\text { Final temperature of } \\
\text { the mixture, }{ }^{\mathbf{O}} \mathbf{C}\end{array}$ & $\begin{array}{c}\text { Time of } \\
\text { mixing, min }\end{array}$ & $\begin{array}{c}\text { Energy } \\
\text { consumption, kWh }\end{array}$ \\
\hline $\begin{array}{c}\text { NMC dilution with chilled water } \\
\text { in the fermenter }\end{array}$ & 28.3 & 30.1 & 0.060 \\
\hline $\begin{array}{c}\text { NMC mixing with water in the } \\
\text { mixer }\end{array}$ & 37.3 & 14.3 & 0.025 \\
\hline
\end{tabular}

The dynamics of the temperature change in the fermenter (Fig. 3) shows that jet mixing, unlike the standard scheme, does not imply sharp temperature changes inside the fermenter.

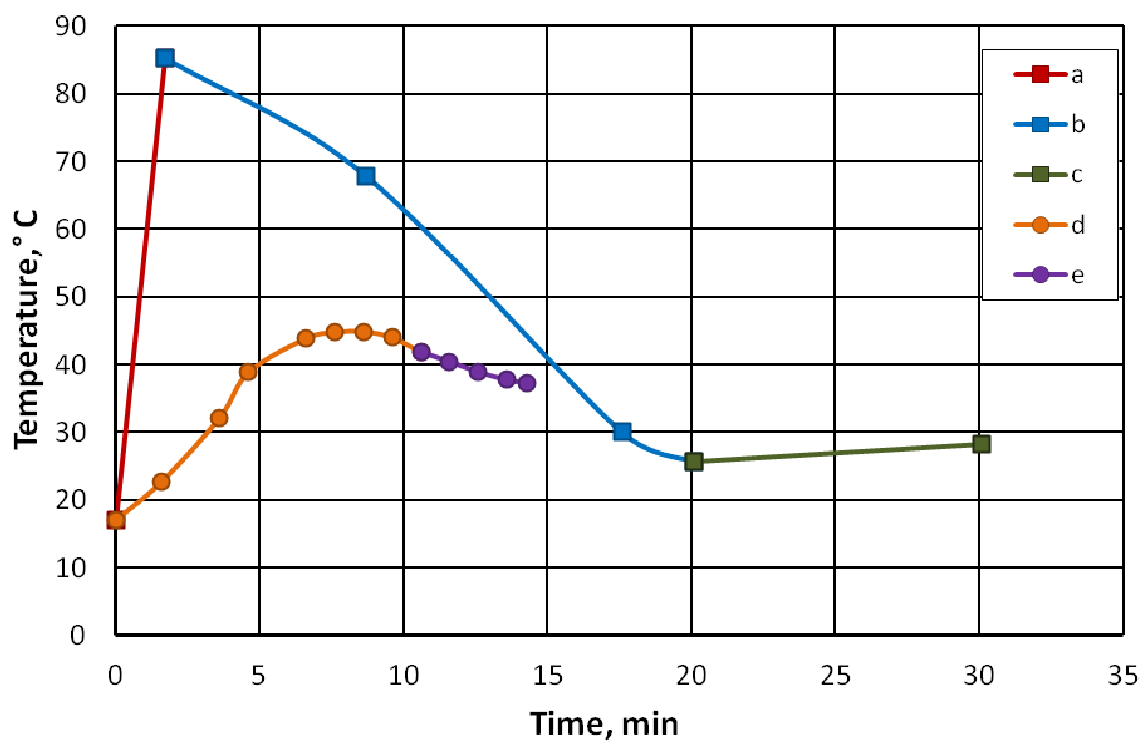

Fig. 3. Dynamics of the temperature change of the mixture in the fermenter: the standard mixing scheme ( $a$ - the supply of the NMC from the steriliser to the fermenter; $b$ - dilution of the NMC with water; $\mathrm{c}$ - homogenisation of the mixture with a mixer of the fermenter); the new scheme of mixing

( $\mathrm{d}$ - jet mixing of the NMC with water; $\mathrm{e}$ - dilution of the mixture with water in the fermenter)

Also, during the tests it was found that exact dosage of the volumes of the mixed media, using JS, requires refinement. This is evidenced by the stage of dilution of the mixture with water (section e, Fig. 3) in the absence of the nozzle flow, since, by this moment, the entire volume of the NMC was already pumped out of the steriliser. The averaged flow parameters in front of the mixer are given in Table 2. The final answer about the efficiency of using different mixing schemes can be given only by experiments with the preparation of NM and the subsequent cultivation of microorganisms. However, considering the results of the preliminary tests, we can say that the use of jet mixing will significantly reduce the energy consumption for preparation of the NM and reduce the time of this process, compared to the sequential supply of NMC and water to the fermenter.

Table 2

Flow parameters in front of the JM

\begin{tabular}{|c|c|c|c|c|c|c|c|c|}
\hline \multirow{2}{*}{$\begin{array}{c}\text { Diameter of } \\
\text { the nozzle, } \\
\text { mm }\end{array}$} & \multirow{2}{*}{$\begin{array}{l}\text { Diameter } \\
\text { of the tee- } \\
\text { joint, } \mathrm{mm}\end{array}$} & \multicolumn{2}{|c|}{ Pressure, bar } & \multicolumn{2}{|c|}{$\begin{array}{c}\text { Consumption, } \\
1 \cdot \min ^{-1}\end{array}$} & \multicolumn{2}{|c|}{$\begin{array}{c}\text { JS inlet } \\
\text { temperature, }{ }^{\circ} \mathrm{C}\end{array}$} & \multirow{2}{*}{$\begin{array}{c}\text { Final } \\
\text { temperature of } \\
\text { the mixture, }{ }^{\circ} \mathrm{C}\end{array}$} \\
\hline & & NMC & water & NMC & water & NMC & water & \\
\hline 2 & 15 & 1.75 & 0.08 & 1.54 & 4.52 & 98 & 11 & 37 \\
\hline
\end{tabular}

\section{Conclusions}

There is substantiated the expediency of using jet mixing of the components as the most energyefficient technology for the preparation of nutrient media from liquid components that have undergone separate sterilisation. A simple jet mixer type on the base of a standard pipe tee was proposed for the 
mixing process. The jet mixer tests showed the possibility of mixing time reduction by half from 30 min to $14 \mathrm{~min}$. This led to proportional power consumption decrease from $60 \mathrm{Wh}$ for the standard technique of nutrient components supplying to the fermenter and further mixture homogenizing by agitator to $25 \mathrm{Wh}$ for jet mixing of the components. Jet mixing at the same time showed much less temperature difference inside the fermenter $\left(25^{\circ} \mathrm{C}\right)$, compared with the standard processing technique, which showed more than $55^{\circ} \mathrm{C}$ difference. Within the framework of further research, it is expedient to consider the possibilities of integrated use of the jet mixers in technological processes for production of microbiological preparations at the fermentation complex that we have developed.

\section{References}

[1] Starchevskyj JuI., Dubrovin V. To the issue of the development of regional biofactory and biological laboratory network for biological agriculture. Scientific Journal of NULES of Ukraine. 2011; vol.166(1), pp. 43-55.

[2] Bespalov I., Hodorchuk V. Economic fermentative unit for production of microbiological means of protections of plants. Bulletin of Agricultural Science. 2017, Vol.1, pp. 38-42.

[3] Yaroshevsky V. P., Krutyakova V. I. Thin-walled fermentation complexes energy efficiency improvement. Engineering and technology innovations in the producing of entomological control agents and microbial pesticides for plant protection. 2017, Odesa, pp.175-89.

[4] Ovcharuk V. S, Melnychuk A. A, Kosoj S. M., Melnyk P. I., Starchevskyj Jul. Thin-walled fermenter. Patent UA 82696, IPC A61L 2/04, C12M 1/12. 2008.

[5] Stanbury P., Whitaker A., Hall S. Principles of fermentation technology. $3^{\text {rd }}$ ed. Oxford, Butterworth-Heinemann Ltd; 2016, 824 p.

[6] Bailey JE, Ollis DF. Biochemical engineering fundamentals. $2^{\text {nd }}$ ed. New York. McGraw-Hill Education. 1986, 984 p.

[7] Lyamaev B. F. Liquid-Jet Pumps and Facilities. Leningrad, Mashinostroenie, 1988, 256 p.

[8] Wilman J. T. Jet pumps. 1966, Brussels: Guyot, 64 p.

[9] Pranav K, Ranjan HK, Singh MP. A study and analyze of an ejector in steam power plant. International Journal of Engineering and Management Research. 2014, vol.4(3), pp. 195-199.

[10] Samoychuk K., Poludnenko O. Results of analysis of constructions of stream mixers of liquid components. Pratsi Tavriyskogo derzhavnogo agrotehnologichnogo universitetu. 2013. 13 (1). pp. 205-211.

[11] Chapman T.C., Terry J.C. Water aerator and method of using same. Pateent US 6986506 Int. Cl. B01F 3/04, 2006.

[12] La Crosse G.R.Aerator and wastewater treatment system. Patent US 6682057. Int. Cl. B01F 3/0, 2004. 